\title{
Study of the association of the T869C polymorphism of the transforming growth factor- $\beta 1$ gene with polycystic ovary syndrome
}

\author{
JUNG-HYUN PARK, LAN LI and KWANG-HYUN BAEK \\ Department of Biomedical Science, CHA University, Bundang CHA Hospital, \\ Seongnam-Si, Gyeonggi-Do 463-840, Republic of Korea
}

Received August 8, 2014; Accepted April 30, 2015

DOI: $10.3892 / \mathrm{mmr} .2015 .3896$

\begin{abstract}
Polycystic ovary syndrome (PCOS) is a common multifactorial disorder characterized by hyperandrogenism, insulin resistance and chronic oligoanovulation. In addition, a number of females with PCOS have ovaries with multiple cysts, an irregular or no menstrual cycle and an imbalance of female hormones compared with normal controls. The transforming growth factor $\beta 1$ (TGF- $\beta 1)$ gene is one of the genes associated with obesity and type 2 diabetes, which are characteristic symptoms of PCOS. The present study, therefore, investigated the association between the T869C polymorphism of the TGF- $\beta 1$ gene, a single nucleotide polymorphism (SNP) of TGF- $\beta 1$ and PCOS. The genomic DNA from 285 patients with PCOS and 129 healthy control individuals was used in the present study. $\mathrm{P}<0.05$ was considered to indicate a statistically significant difference between the groups. The present study findings suggested that the frequency of genotypes provided no significant association between the T869C polymorphism in the TGF- $\beta 1$ gene and patients with PCOS. Although the present study concluded that the T869C polymorphism in the $T G F-\beta 1$ gene is not associated with the pathogenesis of PCOS, further studies regarding the correlation between other SNPs of the TGF- $\beta 1$ gene and PCOS are required.
\end{abstract}

\section{Introduction}

Polycystic ovary syndrome (PCOS) is a complex syndrome and is the most common endocrine disorder in females of reproductive age globally (1). In a previous study, $4-4.8 \%$ of white females and $3.5 \%$ of African females exhibited PCOS (2). Similarly, a previous study demonstrated that

Correspondence to: Professor Kwang-Hyun Baek, Department of Biomedical Science, CHA University, Bundang CHA Hospital, 335 Pangyo-Ro, Seongnam-Si, Gyeonggi-Do 463-840, Republic of Korea

E-mail: baek@cha.ac.kr

Key words: polycystic ovary syndrome, single nucleotide polymorphism, transforming growth factor $\beta 1$ the prevalence of PCOS was $4.9 \%$ among female college students in Korea (3). According to the American Society for Reproductive Medicine (ASRM) and the European Society of Human Reproduction and Embryology (ESHRE), PCOS is diagnosed when the phenotype of patients satisfy two of the following three criteria: Oligomenorrhea or amenorrhea, biochemical hyperandrogenism or polycystic ovaries $(4,5)$. Generally, females with PCOS also exhibit symptoms of an increased risk of infertility, obesity, hyperlipidemia, insulin resistance, type 2 diabetes (T2D), diabetic nephropathy (DN) and possibly cardiovascular disease (6-8).

The pathogenesis of PCOS is initiated by various signaling pathways: Metabolism, insulin-signaling, inflammation and angiogenesis (9). Among these pathways, insulin resistance or hyperinsulinemia triggers changes in the hypothalamo-pituitary-ovarian axis, causing excessive production of androgen, leading to aberrant follicular development. Hyperinsulinemia in PCOS inhibits the hepatic sex hormone binding globulin from increasing the levels of free testosterone (T) in the body. This promotes the secretion of luteinizing hormone ( $\mathrm{LH})$ with relatively low follicle-stimulating hormone (FSH) (10). For this reason, it is recognized that females with PCOS are susceptible to insulin resistance and T2D (11). A case-control genetic association investigation regarding the pathogenesis of PCOS focused on the single nucleotide polymorphisms (SNPs) affecting the inflammatory processes and the activity of transforming growth factor- $\beta 1$ (TGF- $\beta 1$ ) (12). In previous years, genetic studies have linked PCOS to a dinucleotide repeat marker, D19S884, in the fibrillin 3 gene. Fibrillins are important molecules, which assemble into microfibrils in the extracellular matrix (ECM) to modulate the $T G F-\beta 1$ signaling pathway $(13,14)$. Therefore, variations in fibrillin 3 and the subsequent dysregulation of TGF- $\beta$ may contribute to the pathogenesis of PCOS (15).

TGF- $\beta$ is a multifunctional cytokine synthesized in a wide variety of tissue types and it is secreted from various cell types (16). The TGF- $\beta$ superfamily consists of three isoforms: $T G F-\beta 1, T G F-\beta 2$ and $T G F-\beta 3$ (17). The members of the human TGF- $\beta$ superfamily are critical modulators in apoptosis and cell survival (18). The TGF- $\beta$ signaling pathway is initiated when a TGF- $\beta$ superfamily ligand binds to a high-affinity transmembrane receptor complex, 
composed of the activin-like kinase $5 / \mathrm{TGF}-\beta$ type 1 receptor and the TGF- $\beta$ type 2 receptor. Each class of ligand binds to a specific type 2 receptor, which has a serine/threonine kinase domain (19). It subsequently recruits and phosphorylates a specific type 1 receptor. The type 1 receptor phosphorylates receptor-regulated Smads, which subsequently bind to the coSmad, Smad4. R-Smad/coSmad complexes activated by phosphorylation translocate into the nucleus to bind to gene promoters and activate the expression of the target genes involved in cell proliferation and differentiation $(20,21)$. This TGF- $\beta$ superfamily signaling demonstrates its potential role in embryonic development, cellular differentiation, hormone secretion and immune system functions $(22,23)$. In addition to this Smad-mediated gene transcription, TGF- $\beta$ signaling is involved in activating Smad-independent pathways, including the nuclear factor- $\kappa \mathrm{B}$ pathway (24), the mitogen-activated protein kinase/ERK pathway (25) and the phosphatidylinositol-3-kinase/Akt signaling pathway (26). Therefore, Smad-independent pathways in the TGF- $\beta$ family signaling pathways have significant effects on the different biological functions of TGF- $\beta$, including cell cycle inhibition, immune suppression and neuroprotective effects $(27,28)$.

The TGF- $\beta$ signaling pathway has a vital role in the development of multiple tissues or cells, including folliculogenesis, which is the process of developing ovarian follicles (29). Members of the TGF- $\beta$ superfamily are expressed in mammalian oocytes and thereby, the subsequent dysregulation of TGF- $\beta$ has been implicated in the pathogenesis of abnormal follicle development and hyperandrogenism in patients with PCOS (30,31). A previous study indicated that the ovaries of females with PCOS exhibited all the markers of increased TGF- $\beta$ activity (32).

The $T G F-\beta 1$ gene has been suggested as a genetic factor due to the clinical symptoms of PCOS, including an increased risk of T2D. TGF- $\beta 1$ promotes the production of the ECM in response to high levels of glucose. Therefore, TGF- $\beta 1$ is considered to be central in the pathogenesis of DN (33).

The human TGF- $\beta 1$ gene is located on chromosome 19q13.1-13.3 and has six known SNPs: C-988A (rs1800820), G-800A (rs1800468), C509T (rs1800469), T869C (rs1800470; Leu10/P.ro10; T29->C), G915C (rs1800471) and C11929T (THr263Ile; rs1800472) $(34,35)$. In a previous study, a statistically significant difference was detected between the control group and Egyptian patients with T2D in the frequencies of the TGF- $\beta 1$ codon 10 (T869C) (36). The present study, therefore, focused on the T869C polymorphism located on exon 1 , which may be one of the candidate genes associated with an increased risk of DN. The present study aimed to determine whether the T869C polymorphism of $T G F-\beta 1$ was associated with PCOS.

\section{Patients and methods}

Study subjects. All individuals were Korean females $(\mathrm{n}=414)$ of which 129 were healthy controls and 285 were patients with PCOS, recruited from the Fertility Center at CHA General Hospital (Seoul, Korea) between 2008 and 2011. The diagnosis of PCOS was based on the criteria proposed by the 2003 ASRM/ESHRE Rotterdam consensus $(4,5)$. The present study was approved by the Gangnam CHA Fertility
Center (Gyeonggi-Do, Korea). Written informed consent was provided by the patient

Phenotypic characterization of all subjects. Basal blood samples were obtained from patients with PCOS and the controls to measure the levels of the following: Plasma FSH, $\mathrm{LH}$, estrogen (E2), prolactin (PRL), thyroid stimulating hormone (TSH), dehydroepiandrosteronesulphate (DHEAS), $\mathrm{T}$, fasting glucose and insulin.

DNA extraction and genetic analysis. Blood samples were collected in tubes containing EDTA as an anti-clotting factor and stored at $4{ }^{\circ} \mathrm{C}$. The genomic DNA was extracted from the blood of patients with PCOS and the controls. Restriction fragment length polymorphism (RFLP) analysis was performed to determine the genotypes for the T869C polymorphism in exon 1 of the TGF- $\beta 1$ gene. The T869C polymorphism was amplified by polymerase chain reaction (PCR) using the following primers: Forward, 5'-GTACCA GATCGCGCCCATCT-3' and reverse, 5'-TAGCCACAGCAT CGGTAGCAG-3', in a total volume of $30 \mu \mathrm{l}$. In the reaction mixture (Solgent, Seoul, Korea), 100 ng genomic DNA was used as a template. The cycling parameters were as follows: Denaturation at $95^{\circ} \mathrm{C}$ for $5 \mathrm{~min}, 30$ cycles at $95^{\circ} \mathrm{C}$ for $40 \mathrm{sec}$, $65^{\circ} \mathrm{C}$ for $40 \mathrm{sec}$ and $72^{\circ} \mathrm{C}$ for $40 \mathrm{sec}$, followed by $72^{\circ} \mathrm{C}$ for 7 min. Following PCR (C1000 Thermal cycler; Bio-Rad Laboratories, Inc., Hercules, CA, USA), the PCR products of 277 bp were digested with MspA1 I (Enzynomics, Daejeon, Korea) for $2 \mathrm{~h}$ at $37^{\circ} \mathrm{C}$ (Fig. 1A). The restricted DNA fragments were electrophoresed on a $2 \%$ agarose gel (Invitrogen Life Technologies, Carlsbad, CA, USA), containing ethidium bromide (Sigma-Aldrich, St. Louis, MO, USA) and visualized on a DNA Image Visualizer (SeouLin Bioscience Co., Ltd, Seoul, Korea).

A total of three genotypes were observed in the restricted DNA fragments: A single 277 bp band, indicating homozygosity for the T allele; the presence of two fragments, $253 \mathrm{bp}$ and $24 \mathrm{bp}$, indicating homozygosity for the $\mathrm{C}$ allele; the presence of three fragments, $277 \mathrm{bp}, 253 \mathrm{bp}$ and 24-bp bands, indicating heterozygosity for the $\mathrm{T}$ allele and the $\mathrm{C}$ allele, respectively (Fig. 1B).

Statistics analysis. Statistical analysis for comparing the genotype frequencies of the control group and the patient group was performed using Hap analysis (HapAnalyzer Ver.1 0.1, NGRI, Seoul, Korea) and the $\chi^{2}$ test. $\mathrm{P}<0.05$ was considered to inidcate a statistically significant difference.

\section{Results}

Patient characteristics. The 2003 ASRM/ESHRE Rotterdam Consensus was followed to obtain the diagnostic criteria for PCOS. In accordance with these criteria, 285 patients with PCOS were diagnosed when they exhibited at least two of the following three symptoms: Oligomenorrhea or amenorrhea, clinical or biochemical hyperandrogenism and ultrasonographic polycystic ovarian morphology. In the present study, the control group had regular menstrual cycles and no characteristics based on the criteria proposed by the 2003 ASRM/ESHRE Rotterdam consensus. Conversely, the PCOS 
A

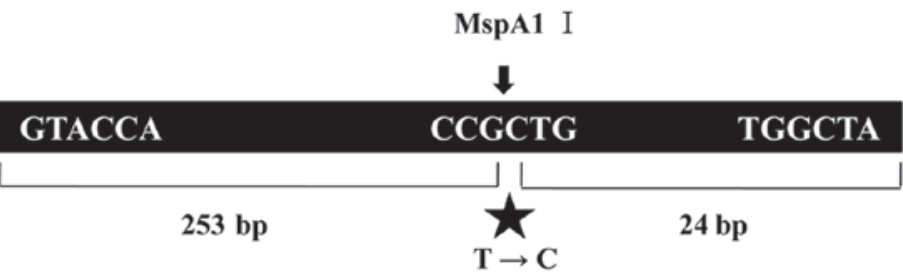

277 bp : Homozygosity for T allele

$253 \mathrm{bp}$ and $24 \mathrm{bp}$ : Homozygosity for C allele

$277 \mathrm{bp}, 253 \mathrm{bp}$ and $24 \mathrm{bp}$ : Heterozygosity for T and C alleles

B

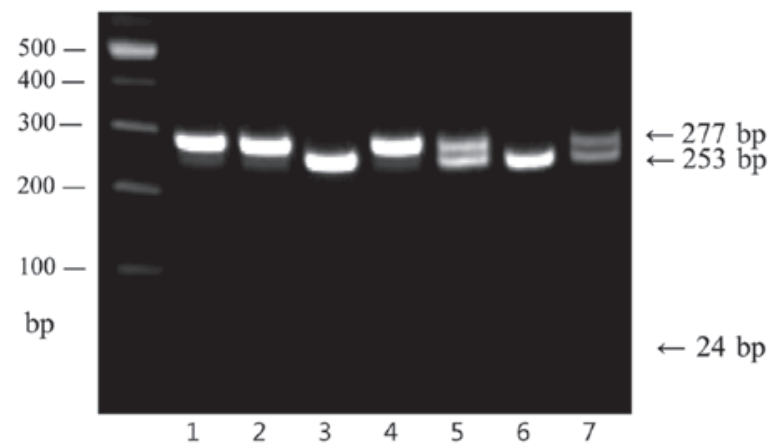

Lane $1:$ TT

Lane $2:$ TT

Lane $3: \mathrm{CC}$

Lane $4:$ TT

Lane $5:$ TC

Lane $6: \mathrm{CC}$

Lane $7:$ TC

Figure 1. (A) Structure of the amplified T869C polymorphism of the TGF- $\beta 1$ gene. An arrow indicates the restriction site of $M s p A 1 \mathrm{I}$. (B) The T/C polymorphism in the $T G F-\beta 1$ gene. The T/T genotype is indicated by a single band at $277 \mathrm{bp}$, the $\mathrm{C} / \mathrm{C}$ genotype is indicated by two bands at 253 and $24 \mathrm{bp}$, and the $\mathrm{T} / \mathrm{C}$ genotype has three bands at 277,253 and $24 \mathrm{bp}$.

Table I. Comparison of disorders and symptoms between the normal controls and patients with PCOS.

\begin{tabular}{lcc}
\hline Characteristic & Controls, $\mathrm{n}=129$ & PCOS patients, $\mathrm{n}=285(\%)$ \\
\hline Hyperandrogenism and oligo- or amenorrhea & 0 & $50(17.54)$ \\
Hyperandrogenism and polycystic ovaries & 0 & $48(16.84)$ \\
Oligo- or amenorrhea and polycystic ovaries & 0 & $143(50.18)$ \\
Hyperandrogenism, oligo- or amenorrhea and polycystic ovaries & 0 & $44(15.44)$
\end{tabular}

PCOS, polycystic ovary syndrome.

Table II. Clinical and biochemical characteristics of normal controls and patients with PCOS.

\begin{tabular}{lccc}
\hline Characteristic & Controls $(\mathrm{n}=129)$ & PCOS patients $(\mathrm{n}=285)$ & P-value \\
\hline BMI $\left(\mathrm{kg} / \mathrm{m}^{2}\right)$ & $20.74 \pm 2.42(16.39-32.56)$ & $22.53 \pm 3.45(16.67-28.02)$ & NS \\
Waist $/$ hip ratio & $0.78 \pm 0.05(0.70-0.98)$ & $0.80 \pm 0.06(0.68-1.09)$ & NS \\
FSH levels $(\mathrm{mIU} / \mathrm{ml})$ & $7.35 \pm 2.02(3.05-20.67)$ & $6.42 \pm 1.89(2.64-18.86)$ & NS \\
LH levels $(\mathrm{mIU} / \mathrm{ml})$ & $3.30 \pm 1.64(0.82-7.03)$ & $6.99 \pm 5.44(1.20-20.08)$ & $<0.001$ \\
E2 levels $(\mathrm{pg} / \mathrm{ml})$ & $32.38 \pm 15.02(5.06-63.38)$ & $41.37 \pm 17.89(8.01-86.36)$ & NS \\
Prolactin levels $(\mathrm{ng} / \mathrm{ml})$ & $12.24 \pm 6.47(4.04-46.29)$ & $13.15 \pm 9.36(2.30-71.54)$ & NS \\
TSH levels $(\mu \mathrm{IU} / \mathrm{ml})$ & $1.82 \pm 0.83(0.04-4.05)$ & $2.30 \pm 1.25(0.42-11.20)$ & NS \\
DHEAS levels $(\mu \mathrm{g} / \mathrm{dl})$ & $148.98 \pm 54.85(65.84-252.45)$ & $178.92 \pm 67.45(48.33-380.1)$ & 0.01 \\
Testosterone $(\mathrm{ng} / \mathrm{ml})$ & $0.22 \pm 0.14(0.02-0.53)$ & $0.43 \pm 0.24(0.07-0.85)$ & $<0.001$ \\
\end{tabular}

BMI, body mass index; FSH, plasma follicular stimulating hormone; LH, lutenizing hormone; E2, estrogen; TSH, thyroid stimulating hormone; DHEAS, dehydroepiandrosteronesulphate; NS, not significant. 
Table III. Genotypes of the T869C polymorphism of transforming growth factor- $\beta 1$ gene in control group and patients with PCOS.

\begin{tabular}{lcc}
\hline Genotype & Control group n $(\%)$ & PCOS patients group n (\%) \\
\hline TT & $41(31.78)$ & $78(27.36)$ \\
TC & $60(46.51)$ & $148(51.92)$ \\
CC & $28(21.71)$ & $59(20.7)$ \\
Total & 129 & 285 \\
\hline
\end{tabular}

PCOS, polycystic ovary syndrome.

patient group revealed that 50 patients $(17.54 \%)$ had hyperandrogenism and oligomenorrhea or amenorrhea, 48 patients (16.84\%) had hyperandrogenism and polycystic ovaries, 143 patients $(50.18 \%)$ had oligomenorrhea or amenorrhea and polycystic ovaries and 44 patients (15.44\%) had hyperandrogenism, oligomenorrhea or amenorrhea and polycystic ovaries (Table I).

Clinical and biochemical features. The clinical and biochemical features of the patients with PCOS and the control group are described in Table II, which demonstrated the body mass index (BMI), waist/hip ratio, obesity and hormone levels, including FSH, LH, E2, PRL, TSH, DHEAS and T. No particular differences were observed between the clinical and biochemical characteristics of the normal controls and the patients with PCOS. However, high levels of LH and T were observed in the PCOS patients, and the DHEA-S level was also slightly higher compared with the control group (Table II).

T869C polymorphism and PCOS. The homozygosity of the $\mathrm{T}$ allele of the $\mathrm{T} 869 \mathrm{C}$ polymorphism in the $T G F-\beta 1$ gene was confirmed by cutting with the MspAl I restriction enzyme (CCGTTG), resulting in the presence of a single fragment of 277-bp. The result of the RFLP analysis demonstrated that the frequencies of three genotypes were present in the T869C polymorphism of the $T G F-\beta 1$ gene. As shown in Table III, the frequency of the T/T, T/C and C/C genotypes in the control and PCOS patient groups demonstrated similar proportions: The rate of the $\mathrm{T} / \mathrm{T}$ genotype was observed in 41 controls $(31.78 \%)$ and 78 patients with $\mathrm{PCOS}(27.36 \%)$, the rate of the T/C genotype was observed in 60 controls $(46.51 \%)$ and 148 patients with PCOS $(51.92 \%)$, and the rate of the $\mathrm{C} / \mathrm{C}$ genotype was observed in 28 controls $(21.71 \%)$ and 59 patients with PCOS (20.7\%; Table III). These results revealed no significant association between the T869C polymorphism in the TGF- $\beta 1$ gene and the patients with PCOS.

\section{Discussion}

TGF- $\beta$ is a multifunctional cytokine, which exerts its biological function by regulating several cellular processes, including proliferation, differentiation, embryonic development, ECM formation, angiogenesis and immunity (37). Altered expression of $T G F-\beta 1$ due to polymorphisms exerts an affect on numerous normal cellular and disease processes, including T-cell activation and proliferation, tumor development, and asthma (38). Among the $T G F-\beta 1$ polymorphisms, the polymorphism at codon 10 (T869C) may be associated with higher or lower $T G F-\beta 1$ synthesis in vitro and may affect a variety of autoimmune-associated diseases, including rheumatoid arthritis, asthma, systemic lupus erythematous and infectious diseases (39). In addition, TGF- $\beta$ is known as an important mediator in ECM molecule production, including fibronectins, collagens and proteoglycans (40). Its overexpression is one of the most continuous molecular characteristics of pathological tissue fibrosis, which leads to multiple organ failure, including the skin, liver, lung and kidney (41). The SNP in codon 10 of $T G F-\beta 1$ changes the amino acid sequence and affects the levels of $T G F-\beta 1$. For this reason, the increased and thickened ovarian stroma of patients with PCOS, which is caused by increasing fibrous tissue and collagen deposition, are signs of the dysregulation of the local TGF- $\beta$ superfamily members and its signaling pathway (42).

Previous studies have suggested the direct effects of TGF- $\beta$ dysregulation on females with PCOS $(43,44)$. Ovarian folliculogenesis is regulated by a balance between extra- and intra-ovarian factors. An imbalance between extra- and intra-ovarian factors results in aberrant folliculogenesis and oogenesis disorder. Intra-ovarian factors include epidermal growth factor, fibroblast growth factors, the insulin-like growth factor family, the neurotrophin growth factor family, the TGF- $\beta$ family, the vascular endothelial growth factor family, the cytokine family and other microenvironmental factors (6). The TGF- $\beta$ superfamily members expressed in the ovary lead to the pathogenesis of anovulation, hyperandrogenism and abnormal follicle development in females with PCOS (30). Furthermore, folliculogenesis and follicle maturation are a series of complicated processes in which mature follicles are differentiated from primordial follicles. This developmental process can be interfered with by aberrant extra-ovarian factors, resulting in ovarian malfunction. These abnormal extra-ovarian endocrine disorders, including FSH deficiency, LH hypersecretion, hyperandrogenism and hyperinsulinemia with insulin resistance, are involved in the pathogenesis of PCOS (45). It has been reported that the majority of patients with PCOS have susceptibility to obesity and T2D. These diseases are caused by the abnormal expression of target genes in patients with PCOS. Of the target genes, the aberrant expression of TGF- $\beta 1$, a significant protein in the insulin signaling pathway, results in $\mathrm{T} 2 \mathrm{D}$, which has symptoms, including glucose tolerance and insulin resistance. In addition, increased levels of $T G F-\beta 1$ in the serum are 
associated with increased IL-1Ra, an anti-inflammatory cytokine. Additionally, increased concentrations of IL-1Ra develop the metabolic regulation of patients with T2D.

It has been reported that an increase in the TC and CC genotype frequency in $T G F-\beta 1$ codon 10 -gene polymorphisms was statistically significant in patients with $\mathrm{T} 2 \mathrm{D}$, and an increased frequency of the TT genotype was significant in the controls. However, several studies investigated the association between the TGF- $\beta 1$ codon 10 gene polymorphism and T2D in the Polish and Chinese populations (46,47). The results of these previous studies revealed different frequencies of genotypes and alleles compared with those of Egyptians (36). This difference between the two previous studies of different ethnic groups may be derived from variations in the allele frequency of the different populations.

The development of low-grade chronic inflammation and the innate immune system, which regulates the effects of genes, fetal programming and metabolic syndrome are significantly involved in the pathogenesis of T2D. Since TGF- $\beta 1$ is a central mediator of the immune system through its primary immunosuppressive effect, it is a critical anti-inflammatory immune regulator (48). $T G F-\beta 1$ also affects $\mathrm{T}$ cells by inhibiting the activation of macrophages. Although the role of the TGF- $\beta$ family in the pathogenesis of PCOS remains to be fully elucidated, reproductive abnormalities appear in knockout mice, which lose function at all levels of the TGF- $\beta$ signaling pathway.

This is the first study, to the best of our knowledge, on the association between the SNP of the $T G F-\beta 1$ gene and patients with PCOS. A previous study reported that the T869C polymorphism of TGF- $\beta 1$ is associated with T2D and obesity (35), however the authors did not analyze its association with PCOS. In the present study, the results revealed no significant correlation between the T869C polymorphism in the TGF- $\beta 1$ gene and females with PCOS. Therefore, this present genetic association study indicated no evidence of the involvement of the $\mathrm{T} 869 \mathrm{C}$ polymorphism in the TGF- $\beta 1$ gene in PCOS. However, further genotypic association investigations into other SNPs of the TGF- $\beta 1$ gene and PCOS are required. In addition, investigations regarding the TGF- $\beta 1$ gene and patients of different ethnic groups with PCOS are required.

\section{Acknowledgements}

The authors would like to thank the members of the Fertility Center and Stem Cell Institute at CHA University and CHA General Hospital. This study was supported by a grant from the Brain Korea 21 (BK21) PLUS project in Korea.

\section{References}

1. Zadeh-Vakili A, Ramezani Tehrani F, Daneshpour MS, Zarkesh M, Saadat N and Azizi F: Genetic polymorphism of vitamin D receptor gene affects the phenotype of PCOS. Gene 515: 193-196, 2013.

2. Carmina E and Lobo RA: Polycystic ovary syndrome (PCOS): arguably the most common endocrinopathy is associated with significant morbidity in women. J Clin Endocrinol Metab 84: 1897-1899, 1999.

3. Futterweit W: Polycystic ovary syndrome: Clinical perspectives and management. Obstet Gynecol Surv 54: 403-413, 1999.
4. Rotterdam ESHRE/ASRM-Sponsored PCOS Consensus Workshop Group: Revised 2003 consensus on diagnostic criteria and long-term health risks related to polycystic ovary syndrome. Fertil Steril 81: 19-25, 2004.

5. Rotterdam ESHRE/ASRM-Sponsored PCOS Consensus Workshop Group: Revised 2003 consensus on diagnostic criteria and long-term health risks related to polycystic ovary syndrome (PCOS). Hum Reprod 19: 41-47, 2004.

6. Qiao J and Feng HL: Extra- and intra-ovarian factors in polycystic ovary syndrome: impact on oocyte maturation and embryo developmental competence. Hum Reprod Update 17: 17-33, 2011.

7. Tal R, Seifer DB, Shohat-Tal A, Grazi RV and Malter HE: Transforming growth factor-betal and its receptor soluble endoglin are altered in polycystic ovary syndrome during controlled ovarian stimulation. Fertil Steril 100: 538-543, 2013.

8. Diamanti-Kandarakis E, Kandarakis H and Legro RS: The role of genes and environment in the etiology of PCOS. Endocrine 30: 19-26, 2006.

9. Dantas WS, Gualano B, Rocha MP, Barcellos CR, dos Reis Vieira Yance V and Marcondes JA: Metabolic disturbance in PCOS: clinical and molecular effects on skeletal muscle tissue. Scientific World J 2013: 178364, 2013.

10. Doi SA, Towers PA, Scott CJ and Al-Shoumer KA: PCOS: an ovarian disorder that leads to dysregulation in the hypothalamic-pituitary-adrenal axis? Eur J Obstet Gynecol Reprod Biol 118: 4-16, 2005.

11. Ehrmann DA: Metabolic dysfunction in pcos: Relationship to obstructive sleep apnea. Steroids 77: 290-294, 2012.

12. Luque-Ramírez M, San Millán JL and Escobar-Morreale HF: Genomic variants in polycystic ovary syndrome. Clin Chim Acta 366: 14-26, 2006.

13. Urbanek M, Woodroffe A, Ewens KG, et al: Candidate gene region for polycystic ovary syndrome on chromosome 19p13.2. J Clin Endocrinol Metab 90: 6623-6629, 2005.

14. Stewart DR, Dombroski BA, Urbanek M, et al: Fine mapping of genetic susceptibility to polycystic ovary syndrome on chromosome $19 \mathrm{p} 13.2$ and tests for regulatory activity. J Clin Endocrinol Metab 91: 4112-4117, 2006.

15. Jordan CD, Bohling SD, Charbonneau NL and Sakai LY: Fibrillins in adult human ovary and polycystic ovary syndrome: is fibrillin-3 affected in PCOS? J Histochem Cytochem 58: 903-915, 2010.

16. Letterio JJ and Roberts AB: Regulation of immune responses by TGF-beta. Annu Rev Immunol 16: 137-161, 1998.

17. Govinden R and Bhoola KD: Genealogy, expression and cellular function of transforming growth factor-beta. Pharmacol Ther 98: 257-265, 2003.

18. Taipale J, Saharinen J and Keski-Oja J: Extracellular matrix-associated transforming growth factor-beta: role in cancer cell growth and invasion. Adv Cancer Res 75: 87-134, 1998.

19. Ten Dijke P and Hill CS: New insights into TGF-beta-Smad signalling. Trends Biochem Sci 29: 265-273, 2004.

20. Bachman KE and Park BH: Duel nature of TGF-beta signaling: tumor suppressor vs. tumor promoter. Curr Opin Oncol 17: 49-54, 2005.

21. Moustakas A, Souchelnytskyi S and Heldin CH: Smad regulation in TGF-beta signal transduction. J Cell Sci 114: 4359-4369, 2001.

22. Massagué J: How cells read TGF-beta signals. Nat Rev Mol Cell Biol 1: 169-178, 2000.

23. Shi Y and Massague J: Mechanisms of TGF-beta signaling from cell membrane to the nucleus. Cell 113: 685-700, 2003.

24. König HG, Kögel D, Rami A and Prehn JH: TGF-\{beta\}1 activates two distinct type I receptors in neurons: implications for neuronal NF-\{kappa\}B signaling. J Cell Biol 168: 1077-1086, 2005.

25. Derynck $R$ and Zhang YE: Smad-dependent and Smad-independent pathways in TGF-beta family signalling. Nature 425: 577-584, 2003.

26. Caraci F,Battaglia G, Busceti C, et al: TGF-beta 1 protects against Abeta-neurotoxicity via the phosphatidylinositol-3-kinase pathway. Neurobiol Dis 30: 234-242, 2008.

27. Zhu Y, Culmsee C, Klumpp S and Krieglstein J: Neuroprotection by transforming growth factor-betal involves activation of nuclear factor-kappaB through phosphatidylinositol-3-OH kinase/Akt and mitogen-activated protein kinase-extracellular-signal regulated kinase 1, 2 signaling pathways. Neuroscience 123: 897-906, 2004.

28. Bosco P, Ferri R, Salluzzo MG, et al: Role of the transformin g-growth-factor-beta1 gene in late-onset Alzheimer's disease: implications for the treatment. Curr Genomics 14: 147-156, 2013. 
29. Sproul K, Jones MR, Mathur R, Azziz R and Goodarzi MO Association study of four key folliculogenesis genes in polycystic ovary syndrome. BJOG 117: 756-760, 2010.

30. Welt CK, Taylor AE, Fox J, Messerlian GM, Adams JM and Schneyer AL: Follicular arrest in polycystic ovary syndrome is associated with deficient inhibin A and B biosynthesis. J Clin Endocrinol Metab 90: 5582-5587, 2005.

31. Eldar-Geva T, Spitz IM, Groome NP, Margalioth EJ and Homburg R: Follistatin and activin A serum concentrations in obese and non-obese patients with polycystic ovary syndrome. Hum Reprod 16: 2552-2556, 2001.

32. Hatzirodos N, Bayne RA, Irving-Rodgers HF, et al: Linkage of regulators of TGF-beta activity in the fetal ovary to polycystic ovary syndrome. FASEB J 25: 2256-2265, 2011.

33. Akai Y, Sato H, Ozaki H, Iwano M, Dohi Y and Kanauchi M: Association of transforming growth factor-betal T29C polymorphism with the progression of diabetic nephropathy. Am J Kidney Dis 38 (4 Suppl 1): 182-185, 2001.

34. Peng Z, Zhan L, Chen S and Xu E: Association of transforming growth factor-beta1 gene C-509T and T869C polymorphisms with atherosclerotic cerebral infarction in the Chinese: a case-control study. Lipids Health Dis 10: 100, 2011.

35. Jia H, Yu L, Gao B and Ji Q: Association between the T869C polymorphism of transforming growth factor-beta 1 and diabetic nephropathy: a meta-analysis. Endocrine 40: 372-378, 2011.

36. El-Sherbini SM, Shahen SM, Mosaad YM, Abdelgawad MS and Talaat RM: Gene polymorphism of transforming growth factor-beta1 in Egyptian patients with type 2 diabetes and diabetic nephropathy. Acta Biochim Biophys Sin (Shanghai) 45: 330-338, 2013.

37. Hou YL, Chen H, Dong ZH, et al: Clinical significance of serum transforming growth factor-betal in lung cancer. Cancer Epidemiol 37: 750-753, 2013.

38. Shah R, Rahaman B, Hurley CK and Posch PE: Allelic diversity in the TGFB1 regulatory region: characterization of novel functiona single nucleotide polymorphisms. Hum Genet 119: 61-74, 2006.
39. Awad MR, El-Gamel A, Hasleton P, Turner DM, Sinnott PJ and Hutchinson IV: Genotypic variation in the transforming growth factor-betal gene: association with transforming growth factor-beta1 production, fibrotic lung disease and graft fibrosis after lung transplantation. Transplantation 66 : 1014-1020, 1998.

40. Sharma K and Ziyadeh FN: The emerging role of transforming growth factor-beta in kidney diseases. Am J Physiol 266: F829-F842, 1994.

41. Border WA and Noble NA: Transforming growth factor beta in tissue fibrosis. N Engl J Med 331: 1286-1292, 1994.

42. Trombly DJ, Woodruff TK and Mayo KE: Roles for transforming growth factor beta superfamily proteins in early folliculogenesis. Semin Reprod Med 27: 14-23, 2009.

43. Raja-Khan N, Kunselman AR, Demers LM, Ewens KG, Spielman RS and Legro RS: A variant in the fibrillin-3 gene is associated with TGF-beta and inhibin B levels in women with polycystic ovary syndrome. Fertil Steril 94: 2916-2919, 2010.

44. Raja-Khan N, Urbanek M, Rodgers RJ and Legro RS: The role of TGF-beta in polycystic ovary syndrome. Reprod Sci 21: 20-31, 2014.

45. Dumesic DA and Abbott DH: Implications of polycystic ovary syndrome on oocyte development. Semin Reprod Med 26: 53-61, 2008.

46. Buraczynska M, Baranowicz-Gaszczyk I, Borowicz E and Ksiazek A: TGF-beta1 and TSC-22 gene polymorphisms and susceptibility to microvascular complications in type 2 diabetes. Nephron Physiol 106: p69-p75, 2007.

47. Wong TY, Poon P, Chow KM, Szeto CC, Cheung MK and Li PK: Association of transforming growth factor-beta (TGF-beta) T869C (Leu 10Pro) gene polymorphisms with type 2 diabetic nephropathy in Chinese. Kidney Int 63: 1831-1835, 2003.

48. Li MO, Wan YY, Sanjabi S, Robertson AK and Flavell RA: Transforming growth factor-beta regulation of immune responses. Annu Rev Immunol 24: 99-146, 2006. 